\title{
Depth sense aesthesiometry: an advance in the clinical assessment of sensation in the hands
}

\author{
JC SMAJE,* DL MCLELLAN \\ From the Wessex Neurological Centre and the University Department of Medicine, Southampton, UK
}

SUMMARY Fingertip depth sense threshold has been examined in fifty normal subjects using the simple pocket aesthesiometer invented by Renfrew. Index fingers possessed the lowest thresholds and little fingers the highest, whilst there were no significant differences between the same fingers of either hand. Sex and age (at least up to 70 years) had no significant influence on depth sense threshold, but thickened skin and low intelligence tended to raise thresholds. Fingertip depth sense thresholds were then compared with the results of conventional sensory testing in fifty patients with sensory symptoms in the hands. The depth sense threshold of affected fingers was more often abnormal than were the results of clinical tests for light touch appreciation, joint position sense and two-point discrimination. Depth sense aesthesiometry is recommended as a simple, sensitive and quantifiable routine technique for the evaluation of sensory disturbance in the hands.

The clinical examination of patients with sensory symptoms is often time-consuming, and the findings are for the most part poorly quantifiable. Renfrew ${ }^{12}$ described a simple, rapid technique of assessing the threshold of depth sensation in the fingertips, which he believed could supplant the usual methods of examining the modalities of sensation mediated by the dorsal columns of the spinal cord. Depth sense aesthesiometry (as he called the technique) is still not widely employed, and the present study was conducted in order to reappraise its value in routine clinical practice. A preliminary study of methodology was followed by a survey of fingertip depth sense, and certain factors affecting it, in 50 normal subjects. (The term "finger" is used throughout this paper to include the thumb.) The results obtained from depth sense aesthesiometry were then compared with those from conventional bedside sensory tests in 50 patients with sensory disturbance in the hands of varying aetiology.

\section{Methods}

The Renfrew Aesthesiometer

Fingertip depth sense was examined using the "Depth Sense Aesthesiometer" devised by Renfrew. ${ }^{1}$ This is a single piece of transparent acrylic $(2 \mathrm{~cm} \times 10 \mathrm{~cm} \times$ $3 \mathrm{~mm}$ ) bearing on one surface a narrow $(2 \mathrm{~mm})$ ridge rising progressively from zero to $1 \mathrm{~mm}$ in height over

*Present address and address for reprint requests: Dr JC Smaje, Department of Clinical Neurophysiology, Walsgrave Hospital, Clifford Bridge Road, Coventry CV2 2DX, UK. Accepted 1 July 1981 a length of $70 \mathrm{~mm}$, which for the present study was provided with a millimeter scale (fig 1A). By drawing the ridged surface of the device lengthwise over the fingertip, starting from the flat end, an indication of the depth at which deformation of the skin was first appreciated could be obtained (depth sense threshold: DST). Subjects were first acquainted with the test, asked to shut their eyes, and to say "yes" immediately they first detected the ridge. Results were recorded in millimeters along the length of the ridge, which could be converted to actual depth as required. Skin temperature was not recorded, as no significant changes in DST or tactile acuity have been noted within the limits usually encountered. ${ }^{13}$

\section{Methodological considerations}

There were several aspects of technique that were thought could influence results obtained with the aesthesiometer. Those considered most important were: position of testing on the fingertip, velocity of drag, direction of drag (for example, in the longitudinal or transverse axes of the finger), and pressure applied. The first two factors were obviated by using the midpoint between fingertip and terminal whorl at all times (fig 1B), and a constant moderate velocity of drag (taking about 5 seconds for the full length). A preliminary study of the right index finger in 30 subjects from among colleagues and patients without sensory disturbance showed, first, that there was in fact no significant difference in DST whether the aesthesiometer was applied longitudinally or transversely (either direction was subsequently used, according to convenience); second, that reading the result from the advance edge of skin contact (rather than the central point) minimised any effect of applying the device with different pressures (figs 1B, 2). Figure 2 also illustrates the natural tendency for the DST to decline slightly during repeated testing; 
(a)

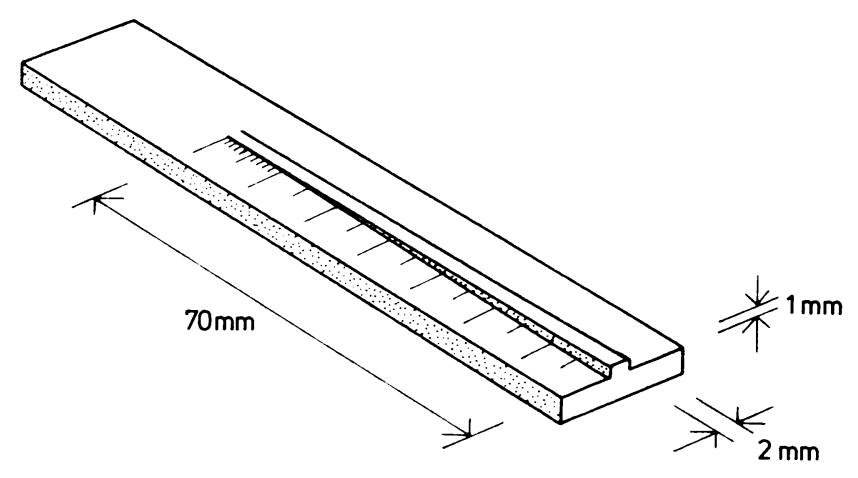

(b)

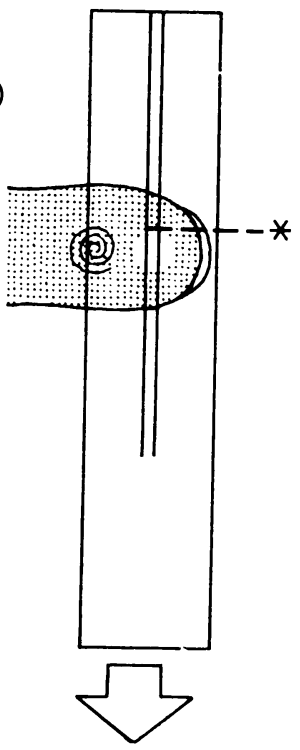

Fig 1 (a) Depth Sense Aesthesiometer as designed by Renfrew, with addition of millimeter scale (applied to surface opposite ridge). Not to scale. (b) Method of using the aesthesiometer, showing its site of application midway between terminal whorl and fingertip, direction of drag (arrow) - the longitudinal axis is equally acceptable, and point at which reading is taken (asterisk). The millimeter scale is omitted for clarity.

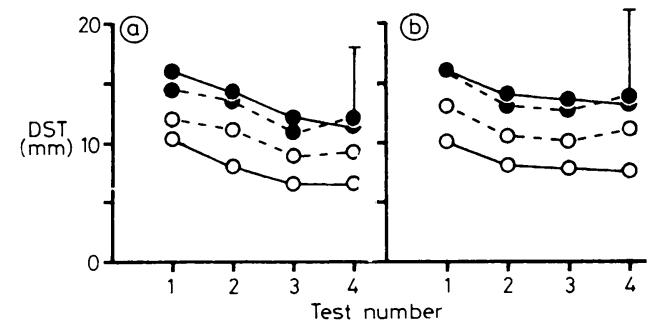

Fig 2 Mean DST readings (in mms along aesthesiometer ridge) from four successive tests on the right index finger of 30 normal subjects. (a) aesthesiometer moved in longitudinal axis of finger, (b) in transverse axis. (O) readings taken at advance edge of skin contact, $(\bigcirc)$ taken at centre point. (-) aesthesiometer pressed firmly, (- -) pressed lightly. The vertical bars show a representative standard deviation above one of the final mean readings.

all subsequent results were recorded as the lower of the final two out of four consecutive estimations (Renfrew suggested taking the third). It was found essential to draw the instrument across the fingertip in a perfectly straight line, as the threshold for edge detection when the ridge is scraped sideways is much lower. Pressing the aesthesiometer against the fingertip at intervals (rather than dragging it) avoided this possibility, but carried the risk of creating an illusory impression of the ridge when the finger was in fact against the flat part of the device ( 12 out of 30 subjects), and this method was not therefore employed.

Subjects studied and test procedures

(a) Fifty people from among colleagues and patients without sensory disturbance participated in the survey of normal subjects (including some of those involved in the preliminary study). They comprised 25 males and 25 females, divided equally among the following age groups: $20-29,30-39,40-49,50-59$ and $60-83$ years (six subjects spanned the decade 60-69 years, and four were aged 72-83 years). The DST of each finger on both hands was examined in randomised order by one of the authors (JCS) according to the technique described above. Differences in DST between each finger on the same and opposite hands were analysed, as well as the influence of handedness, sex, age, skin thickness and intelligence (both assessed subjectively). Two further observers (DLMcL and our colleague $\mathrm{Dr}$ Kate Dalziel) independently measured the DST of index and little fingers in a proportion of this group, in order to assess observer variation. (b) The patient group comprised 17 males and 33 females, age range 18-74 (mean 49) years, all of whom complained of sensory symptoms in one or both hands. The final diagnoses were: generalised peripheral neuropathy $(N=7)$, discrete peripheral nerve or root lesions $(N=15)$, spinal cord lesions $(N=12)$, intracranial lesions other than parietal cortex $(\mathrm{N}=10)$ and parietal cortex lesions $(\mathrm{N}=6)$. In all patients the index and little fingers of both hands (whether affected or not) were tested 
by JCS for light touch appreciation (wisp of cotton wool at fingertip), joint position sense (distal interphalangeal joint), two-point discrimination (2PD: standard calipers at fingertip), and finally depth sense threshold using the aesthesiometer. Graphaesthesia and stereognosis were also tested in those patients with parietal lesions. DST and 2PD were recorded to the nearest millimeter, whilst the other modalities were judged normal, mildly/moderately/ severely impaired, or absent.

\section{Results}

A SURVEY OF NORMAL SUBJECTS

(1) Differences in DST between fingers on the same and on opposite hands

The table shows the mean \pm SD DST readings (in terms of $\mathrm{mms}$ along the ridge) for each finger of both hands (individual readings were distributed in closely normal fashion). Results from calloused or thickened fingers and from subjects of poor intelligence have been excluded, whilst the table incorporates the remaining data from subjects of both sexes and all ages (see sections A (2) and (3) below for justification). For each hand the index finger threshold is slightly, but significantly $(p \leqslant 0.05$, Student's $t$ test), less than that of the thumb, ring and little fingers. The middle finger thresholds are significantly lower only in the case of right middle vs thumb, and left middle vs little finger $(p=0.05)$. There are no significant differences between the remainder. Although the upper limits from left hand fingers lie one to two millimeters higher than those from the right, the differences between their means does not reach statistical significance $(p>0.05)$. This is true also for right-handed and left-handed $(\mathrm{N}=4)$ subjects analysed separately.

\section{(2) Influence of sex and age}

Averaged DST readings from all the digits of each subject were calculated (again excluding thickened fingers and subjects of low intelligence). The means of these values from males $(\mathrm{N}=22)$ and females $(\mathrm{N}=20)$ were $12.64 \pm 3.00 \mathrm{~mm}$ and $11.80 \pm$ $2.66 \mathrm{~mm}$, respectively. This small difference of less than a millimeter (equivalent in actual depth to
$0.012 \mathrm{~mm}$ ) is not significant. The mean values for all subjects in each age group were: $12.54 \pm 3.24 \mathrm{~mm}$ (20-29 yr; $\mathrm{N}=10), 11.92 \pm 3.06 \mathrm{~mm}$ (30-39 yr; $\mathrm{N}=8), \quad 12 \cdot 14 \pm 3.39 \mathrm{~mm} \quad(40-49 \quad \mathrm{yr} ; \quad \mathrm{N}=8)$, $11.55 \pm 3.00 \mathrm{~mm}(50-59 \mathrm{yr} ; \mathrm{N}=8)$ and $12.96 \pm$ $1.60 \mathrm{~mm}(60-83 \mathrm{yr} ; \mathrm{N}=8)$. There are no statistically significant differences between any of these groups, nor any clear trend. This analysis assumes that calculation of a mean DST value for all digits does not conceal any independent behaviour of individual fingers, and that the two variables of sex and age are independent of each other.

\section{(3) Influence of skin thickness and intelligence}

Renfrew ${ }^{2}$ expressed the opinion that intelligence had more effect than skin thickness upon DST readings. The present mean value obtained from fingers judged to be thickened and belonging to those of normal intelligence (assessed subjectively) was $15.03 \pm 7.01 \mathrm{~mm}(\mathrm{~N}=29$ fingers, in four males and four females). The difference between this figure and a mean calculated from the normative data of the table, weighted according to the particular digits involved $(12.08 \pm 3.64 \mathrm{~mm})$, just fails to reach significance, largely because of the greater SD of the former. Five individuals (one male, four female) considered to be of low intelligence had 47 normalthickness fingers between them, their mean threshold being $14.13 \pm 5.89 \mathrm{~mm}$; this is again slightly, but not significantly, greater than the adjusted mean for normally intelligent subjects $(12.32 \pm 3.90 \mathrm{~mm})$. However, the highest thresholds encountered belonged to two male labourers of low intelligence, most of whose fingers were thickened, and whose mean threshold for all fingers was $20.00 \pm 7.60 \mathrm{~mm}$. A further four people incapable of understanding the test were perforce excluded from the study.

\section{(4) Observer variation}

Twenty separate estimations of the right index finger DST were made in two normal subjects over the course of a month. The means for each lay toward the opposite limits of normal, but their ranges and

Table Mean depth sense thresholds in terms of $\mathrm{mm}$ along the aesthesiometer ridge for each finger of both hands in normal subjects

\begin{tabular}{|c|c|c|c|c|c|c|c|c|}
\hline \multirow[t]{2}{*}{ Finger } & \multicolumn{4}{|c|}{ Right hand } & \multicolumn{4}{|c|}{ Left hand } \\
\hline & Mean & $\pm S D$ & $\operatorname{Max}$ & $(N)$ & Mean & $\pm S D$ & $\operatorname{Max}$ & $(N)$ \\
\hline $\begin{array}{l}\text { Thumb } \\
\text { Index } \\
\text { Middle } \\
\text { Ring } \\
\text { Little }\end{array}$ & $\begin{array}{l}13 \cdot 11 \\
10.29 \\
11.56 \\
12.98 \\
13.00\end{array}$ & $\begin{array}{l} \pm 3.19 \\
\pm 3.15 \\
\pm 3.07 \\
\pm 3.95 \\
\pm 4.83\end{array}$ & $\begin{array}{l}20 \\
17 \\
18 \\
21 \\
23\end{array}$ & $\begin{array}{l}(38) \\
(38) \\
(41) \\
(42) \\
(42)\end{array}$ & $\begin{array}{l}12 \cdot 29 \\
10 \cdot 52 \\
11 \cdot 76 \\
13 \cdot 26 \\
13 \cdot 85\end{array}$ & $\begin{array}{l} \pm 4.13 \\
\pm 3.33 \\
\pm 4.01 \\
\pm 4.03 \\
\pm 4.91\end{array}$ & $\begin{array}{l}21 \\
18 \\
20 \\
22 \\
24\end{array}$ & $\begin{array}{l}(42) \\
(42) \\
(42) \\
(42) \\
(42)\end{array}$ \\
\hline
\end{tabular}

$(N)$ indicates the number of subjects contributing to each value-see text for explanation of exclusions among the total of 50 subjects examined (N) ind in the mean plus two standard deviations and rounding up. Note, for example, that at 21 mm along the ridge its height above the base plate, ie the depth to which it depresses the skin, is $0.3 \mathrm{~mm}$. 
SDs indicate a high degree of consistency: $5.40 \pm 0.88$, range $4-7 \mathrm{~mm}$ (34-year-old female); $14 \cdot 85 \pm 1 \cdot 87$, range $12-18 \mathrm{~mm}$ (45-year-old male).

Thirty-two of the 50 normal subjects were examined by a second observer (right index and little fingers only), and 24 of these were also examined by a third. The mean difference between the first and second observers' findings for both fingers was $+0.44 \pm 3.43 \mathrm{~mm}$ (not significant), and between the first and third observers $+2.54 \pm 6.11 \mathrm{~mm}$ (just significant, $\mathrm{p}=0.05$ ).

\section{(5) Summary and normal limits}

The results presented above suggest that DST is lowest in index fingers and highest in little fingers, whilst there are no statistically significant differences between the two hands, the two sexes, or different age groups from 20 to at least 70 years. However, thickened skin and low intelligence (particularly in combination) tend to raise thresholds. Observer error/subject inconsistency and inter-observer variation appear to be small, especially when compared with the inter-subject variability in DST.

Only index and little fingers have been examined in the clinical part of this study. Taking from the table the higher of right and left handed thresholds plus two standard deviations ( $95 \%$ confidence limit) and rounding up, normal upper limits of $18 \mathrm{~mm}$ and $24 \mathrm{~mm}$ have been calculated for index and little fingers respectively. Thickened fingertips and patients unable to co-operate were avoided.

\section{B COMPARISON OF DEPTH SENSE \\ AESTHESIOMETRY WITH OTHER METHODS OF SENSORY TESTING IN PATIENTS WITH SENSORY DISORDERS}

Figures 3 to 5 present the data collected from all 193 fingers successfully examined, 131 of which

(a)

(b)

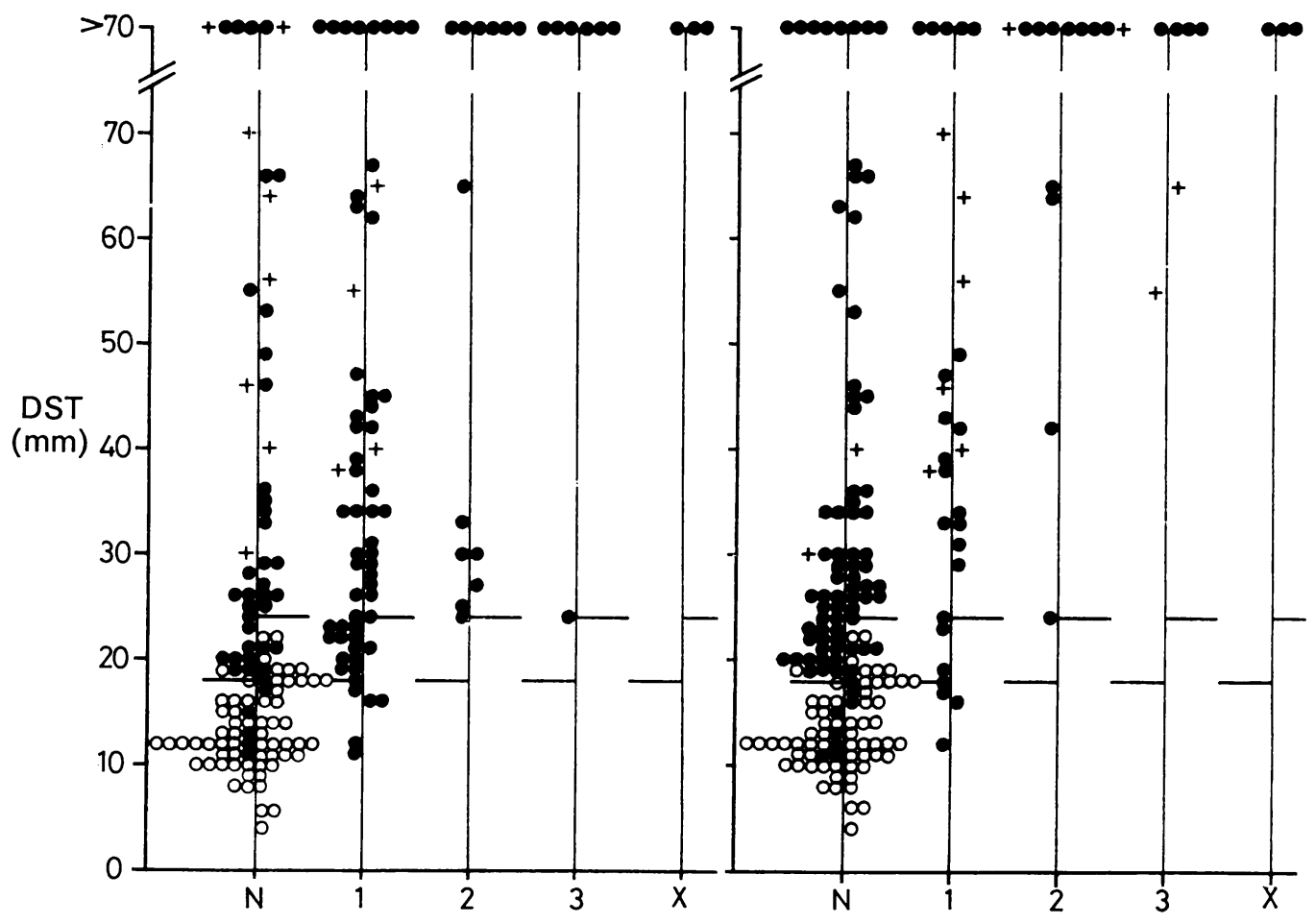

Fig 3 DST readings (in mms along aesthesiometer ridge) from index and little fingers (to left and right of vertical lines, respectively) in 50 patients with sensory symptoms in the hands, compared in the same fingers with (a) light touch appreciation, and (b) joint position sense: $N=$ normal; $1=$ mildly, $2=$ moderately, $3=$ severely impaired; $X=$ absent. The horizontal bars represent upper limits of normal for DST in index fingers (to the left of each vertical line) and little fingers (to the right). (O) readings from symptomatic fingers, $(\bigcirc)$ from asymptomatic fingers, $(+)$ from affected fingers in those patients with parietal cortex lesions. 


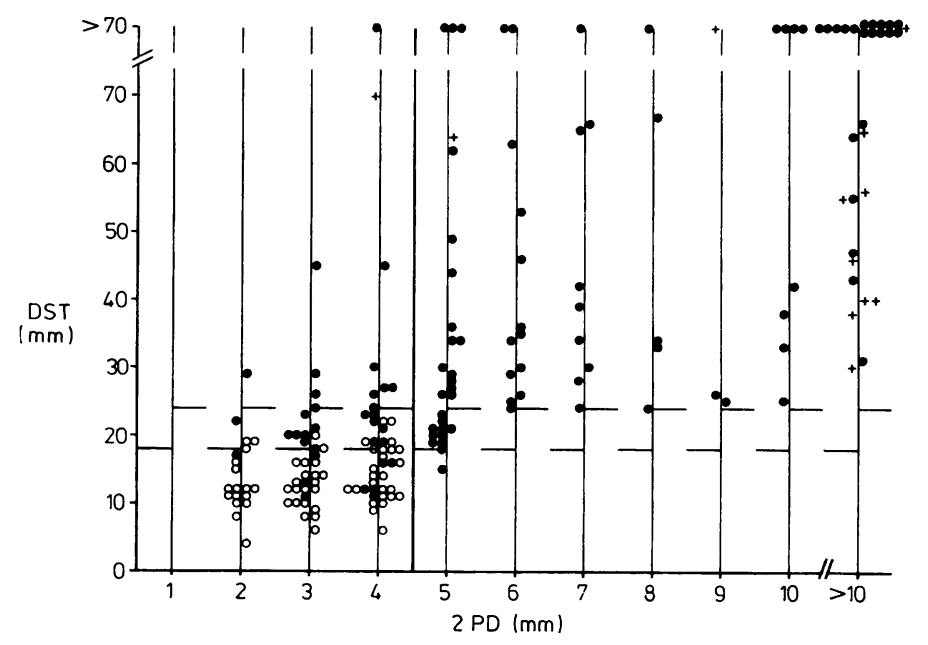

Fig 4 DST readings (in mms along aesthesiometer ridge) from index and little fingers in 50 patients with sensory symptoms in the hands, compared to their 2PD thresholds (to nearest $\mathrm{mm}$ ). The symbols carry the same significance as in fig 3. The vertical bar between 4 and $5 \mathrm{~mm}$ represents the upper limit of normal for $2 P D$.

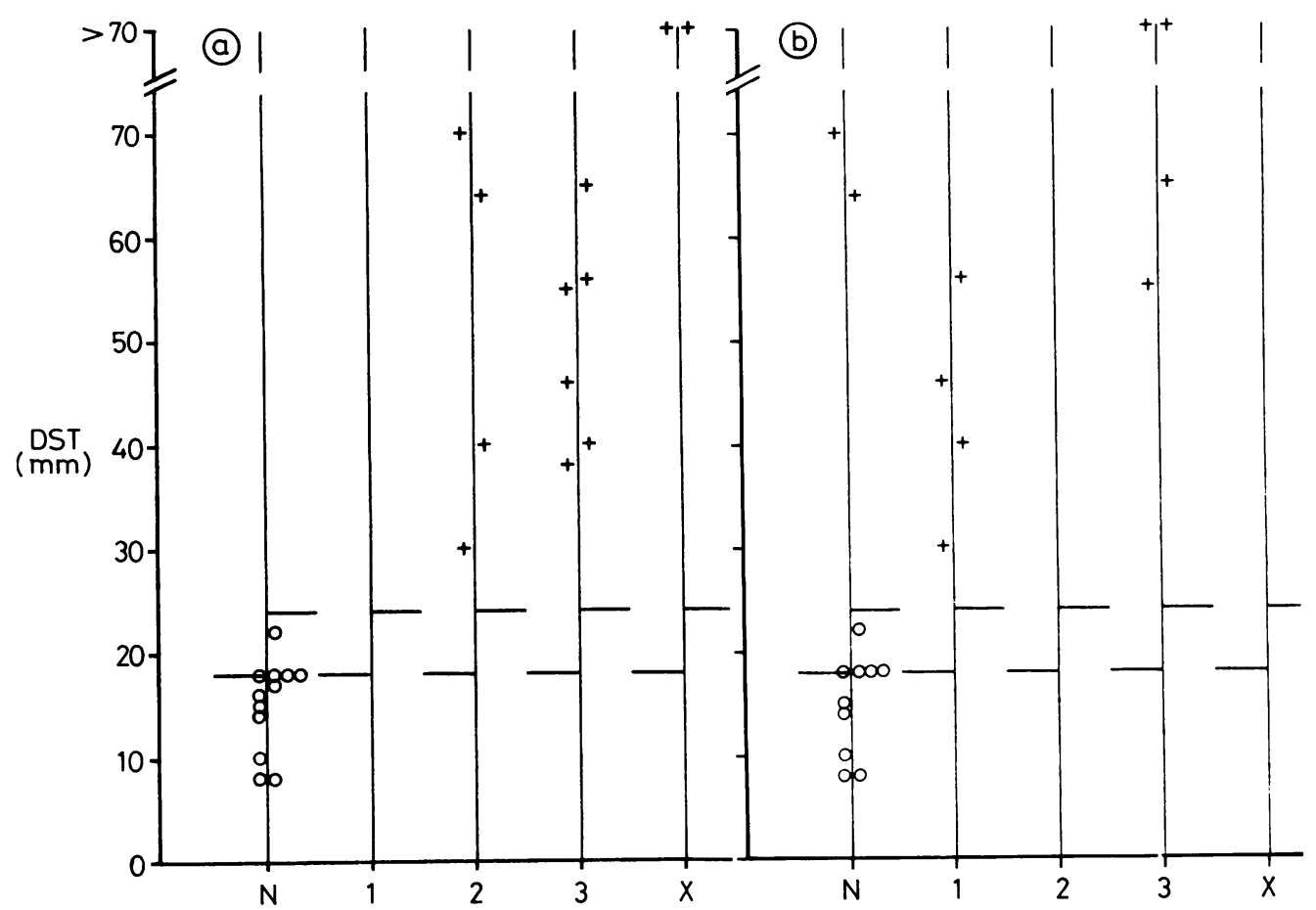

Fig 5 DST readings (in mms along aesthesiometer ridge) from index and little fingers in six patients with parietal cortex lesions, compared in the same hand with (a) graphaesthesia, and (b) stereognosis (one patient untestable). The symbols carry the same significance as in fig 3.

were symptomatic. Figure $3 \mathrm{~A}$ shows that DST fell within normal limits in eight fingers out of a total of 82 exhibiting impaired light touch appreciation. Four out of 51 fingers revealing impaired joint position sense possessed a normal DST (fig 3B). When patients with lesions of the parietal cortex were excluded, 32 out of 41 symptomatic fingers judged to be normal with respect to light touch, and 
65 out of 78 with respect to joint position sense, proved to have abnormal-in some cases markedly abnormal-depth sense thresholds.

Two-PD thresholds ranged from 2 to $4 \mathrm{~mm}$ among unaffected fingers of those patients with clearly localised or lateralised lesions, both for index and little fingers, and $4 \mathrm{~mm}$ was therefore taken to be the highest acceptable value. Figure 4 shows a rough correlation between 2PD and DST, with only a few (two index and one little) fingers showing an abnormal value for the former in the presence of a normal value for the latter. However, 22 out of 35 symptomatic fingers revealed abnormal depth sense when 2PD was within normal limits. Fourteen symptomatic fingers possessed both normal depth sense and 2PD thresholds, five of which were nevertheless found mildly abnormal with regard to light touch appreciation or joint position sense (figs 3,4 ). A single asymptomatic finger displayed a DST above the upper limit (but by only $1 \mathrm{~mm}$ ).

In the few patients found to have parietal cortex lesions, light touch was unimpaired, or only mildly so, whilst joint position sense was rather more often disturbed (fig 3). The remaining modalities were almost always abnormal on the affected side, 2PD and graphaesthesia being generally more severely affected than DST and stereognosis (figs 4,5 ).

\section{Discussion}

When feeling an object with the fingers one's awareness of its size, shape and contour must depend on senses that operate in planes both parallel and perpendicular to the skin. Renfrew and Melville 4 investigated "surface space" and "depth space" thresholds in the hands, using a variety of specially designed test-objects, finding that the absolute values of both increased as one moved proximally from the fingertips, and that they remained in a roughly constant ratio of $3: 1$. However, they drew a distinction between "Depth Space Feeling" and "Touch Feeling Discrimination", by which inference can be made of different levels in an object simply by feeling an edge. The latter has a lower threshold that is similar in finger and palm, and the authors stressed the lack of a clear boundary between the two with regard to depth sense as a whole. The dermal space senses (as represented by 2PD and depth sense) and joint position sense they described together as the "Somatic Sense of Space", or "Choraesthesia", upon which stereognosis and graphaesthesia also depend. Sensory receptors in skin, joint and muscle provide the primary information that subserves these senses, and which is relayed mainly via the dorsal column/medial lemniscus system and thalamus to the somatosensory cortex. The discriminative functions of the space senses depend on the processing of such information that subsequently takes place largely in the parietal cortex. ${ }^{5}$ Which actual sensory endings are concerned with the dermal space senses is still a matter of debate; those involved in two-point discrimination, for example, may not be identical to those responsible for simple touch appreciation. $^{3}$

In evaluating the results of depth aesthesiometry, account must be taken of the naturally different thresholds found between individual fingers (see table). The values quoted here agree closely with those of Renfrew. ${ }^{1}$ Each millimeter along the aesthesiometer ridge corresponds to $0.0143 \mathrm{~mm}$ actual depth; $18 \mathrm{~mm}$ and $24 \mathrm{~mm}$ along the ridge are thus equivalent to approximately $0.26 \mathrm{~mm}$ and $0.35 \mathrm{~mm}$ actual depth. Renfrew gives upper limits of $0.25 \mathrm{~mm}$ and $0.375 \mathrm{~mm}$ for DST in index and little fingers, respectively. Two-PD thresholds have also been reported to be higher in little than in index fingers, ${ }^{6}$ although a recent study surprisingly reached the opposite conclusion. ${ }^{7}$ Taking 2PD thresholds to the nearest millimeter, as is usual in clinical practice, no significant difference in mean 2PD threshold was found between index and little fingers in the present study.

Laterality and sex have both been said to influence tactile and 2PD thresholds, ${ }^{6}$ but these findings are disputed. 78 The present work suggests that for DST the particular hand or sex can, for practical purposes, be ignored.

Whilst no age-related change in DST was found in this study, only four subjects between the ages of 72 and 83 years were examined, a number too small to permit conclusions regarding possible age changes above 70 years. In fact, a decrease in the acuity of several sensory modalities, including $2 \mathrm{PD}$, has frequently been reported in the elderly. 78

The influence of intelligence on DST is more difficult to account for than that of the often occupationally-related thickening of skin. As in other forms of sensory testing, the results depend upon the co-operation of the subject, his ability to understand the test, and his capacity to attend to it. In our experience, subjects found depth sense testing easier than 2PD testing, perhaps because it could be completed more quickly. However, subjects with a prolonged reaction time ${ }^{9}$ might be expected to show falsely raised depth sense thresholds, since the aesthesiometer will have travelled further beyond the threshold point by the time the subject makes his response.

Data were obtained during this study on DST in the toes, but the range of threshold values among normal subjects was much greater than for fingers, often exceeding $70 \mathrm{~mm}$ (1 mm actual depth). 
Scraping the ridge sideways at intervals could be used in this circumstance (when no threshold greater than $35 \mathrm{~mm}$ was found), but the instrument as presently constructed is not suitable for use in sensory testing of the feet.

Clinical observations have shown that the "somatic sense of space" is disturbed by lesions of the peripheral and central pathways and cortical areas outlined above. Examination of at least one aspect of this sense is an important part of neurological assessment. Renfrew ${ }^{2}$ found that, among 100 hands with sensory disturbance, 36 possessed an abnormal DST when 2PD was normal, but the reverse never occurred. From this he concluded that depth aesthesiometry is the more sensitive test, and suggested that when DST is normal it is unnecessary to examine the other less sensitive and poorly quantifiable "choraesthetic" senses. Our findings support this view. Many abnormal DST readings were obtained from symptomatic fingers when other modalities were within normal limits, and rarely vice versa. The diligence with which the conventional sensory tests were performed might perhaps be questioned; equally, such a criticism would serve to emphasise the relative simplicity of depth aesthesiometry.

It must be remembered, of course, that setting DST limits at the $95 \%$ confidence level will allow approximately 1 in 40 readings from normal fingers to be judged abnormally high. Occasional asymptomatic fingers did in fact display unusually high thresholds (four little fingers among controls, one index finger among patients); none was more than $30 \mathrm{~mm}$, and subclinical sensory disturbance in at least some of these cannot be excluded. Nevertheless, the possibility of obtaining occasional false positive findings with the depth aesthesiometer needs to be kept in mind, and confirmation with the usual sensory tests acquired if necessary. A disparity between DST and light touch acuity could alternatively indicate the presence of a discrete parietal cortex lesion, when 2 PD will almost certainly also be abnormal. Raising the upper confidence limit further would probably lead to significant underestimation of increased depth sense thresholds (see fig 4). Even with the upper limits selected, a few false negative findings occurred, but in only three out of 17 such cases was 2PD abnormal.

Since the aesthesiometer has no moving parts, it should rarely need replacing. However, care must be taken in preserving the smooth surfaces of the acrylic ridge, as roughened edges can give rise to spurious low-threshold scraping sensations. When out of use the aesthesiometer surfaces should be protected from scratching.

Although medical students are taught how to test $2 \mathrm{PD}$, most medical wards and health centres in Britain do not possess a pair of calipers, and even in teaching hospitals the sight of a house officer using one is rare indeed. The Depth Sense Aesthesiometer costs much less than 2PD calipers; it is smaller and simpler to use, and more reliable in the exclusion of sensory deficit than other methods. It has the additional advantage of providing a readily quantifiable estimation of sensory threshold. The authors have found it of particular value in the out-patient clinic, and have encouraged the medical students at Southampton University Medical School to familiarise themselves with the technique, in the expectation that some at least will continue to use it in later life.

We are greatly indebted to Dr Stuart Renfrew for a supply of aesthesiometers, and to the consultant staff of the Wessex Neurological Centre for permission to study their patients.

\section{References}

${ }^{1}$ Renfrew S. Aesthesiometers. Lancet 1960;1:1011.

${ }^{2}$ Renfrew S. Fingertip depth sensation-A routine neurological test. Lancet 1969;1:396-7.

${ }^{3}$ Bruce MF, Sinclair DC. The relationship between tactile thresholds and histology in the human finger. $J$ Neurol Neurosurg Psychiatry 1980;43:235-42.

${ }^{4}$ Renfrew S, Melville ID. The Somatic Sense of Space (Choraesthesia) and its threshold. Brain 1960;83: 93-112.

${ }^{5}$ Critchley M. The Parietal Lobes. New York: Hafner, 1953.

${ }^{6}$ Weinstein S. Intensive and extensive aspects of tactile sensitivity as a function of body part, sex and laterality. In: Kenshalo DR, ed. The Skin Senses. Springfield: Thomas, 1968:195-222.

${ }^{7}$ Gellis M, Pool R. Two-point discrimination distances in the normal hand and forearm. Plast Reconstr Surg 1977;59:57-63.

${ }^{8}$ Bruce MF. The relation of tactile thresholds to histology in the fingers of elderly people. J Neurol Neurosurg Psychiatry 1980;43:730-4.

9 Sinclair DC. Psychophysiology of cutaneous sensation. In: Jarrett A, ed. The Physiology and Psychophysiology of the Skin. New York: Academic Press, $1973: 429-73$. 